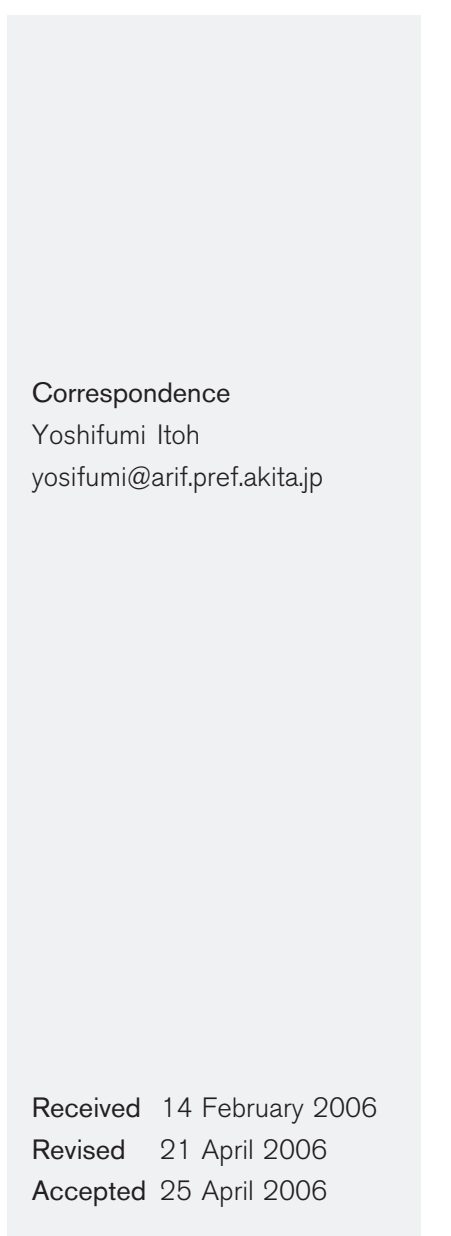

\title{
Characterization and a role of Pseudomonas aeruginosa spermidine dehydrogenase in polyamine catabolism
}

\author{
Veeranki Venkata Dasu, ${ }^{1} \dagger$ Yuji Nakada, ${ }^{1} \ddagger$ Mayumi Ohnishi-Kameyama, ${ }^{1}$ \\ Keitarou Kimura ${ }^{1}$ and Yoshifumi Itoh ${ }^{1,2}$ \\ ${ }^{1}$ National Food Research Institute, Kannondai 2-1-12, Tsukuba, Ibaraki 305-8642, Japan \\ ${ }^{2}$ Akita Research Institute for Food and Brewing, Sanuki 4-26, Araya-machi, Akita 010-1623, \\ Japan
}

\begin{abstract}
Pseudomonas aeruginosa PAO1 has two possible catabolic pathways of spermidine and spermine; one includes the spuA and spuB products with unknown functions and the other involves spermidine dehydrogenase (SpdH; EC 1.5.99.6) encoded by an unknown gene. The properties of SpdH in P. aeruginosa PAO1 were characterized and the corresponding spdH gene in this strain identified. The deduced SpdH (620 residues, calculated $M_{\mathrm{r}}$ of 68861 ) had a signal sequence of 28 amino acids at the amino terminal and a potential transmembrane segment between residues 76 and 92, in accordance with membrane location of the enzyme. Purified SpdH oxidatively cleaved spermidine into 1,3-diaminopropane and 4-aminobutyraldehyde with a specific activity of 37 units (mg protein) $)^{-1}$ and a $K_{\mathrm{m}}$ value of $36 \mu \mathrm{M}$. The enzyme also hydrolysed spermine into spermidine and 3-aminopropanaldehyde with a specific activity of 25 units (mg protein) ${ }^{-1}$ and a $K_{\mathrm{m}}$ of $18 \mu \mathrm{M}$. Knockout of $s p d H$ had no apparent effect on the utilization of both polyamines, suggesting that this gene is minimally involved in polyamine catabolism. However, when $s p d H$ was fused to the polyamine-inducible promoter of $s p u A$, it fully restored the ability of a spuA mutant to utilize spermidine. It is concluded that $\mathrm{SpdH}$ can perform a catabolic role in vivo, but $P$. aeruginosa PAO1 does not produce sufficient amounts of the enzyme to execute this function.
\end{abstract}

\section{INTRODUCTION}

Pseudomonas species, including Pseudomonas aeruginosa, can utilize polyamines (putrescine, spermidine and spermine) as the sole source of carbon and nitrogen ( $\mathrm{Lu}$ et al., 2002; Nishijyo et al., 2001; Stanier et al., 1966). P. aeruginosa PAO1 initially converts putrescine into 4 -aminobutyraldehyde via putrescine:pyruvate aminotransferase, encoded by $s p u C(\mathrm{Lu}$ et al., 2002). This intermediate aldehyde is then oxidized to 4 -aminobutyrate by a dehydrogenase specified by $k a u B$ before being channelled into the TCA cycle (Jann et al., 1988; Fig. 1). The spuC gene is part of the spuABCDEFGH operon, in which spuA and spuB encode putative enzymes with homology to glutamine amidotransferase and glutamine synthetase, respectively, and

†Present address: Department of Biotechnology, Indian Institute of Technology-Guwahati, North Guwahati, Guwahati-781039, Assam, India.

‡Present address: Department of Nursing, Faculty of Nursing and Rehabilitation, Aino University, Higashiohda 4-5-4, Ibaraki, Osaka 5670012, Japan.

Two supplementary figures are available with the online version of this paper. the spuDEFGH cluster specifies a transport system of spermidine (Lu et al., 2002). Expression of the spu operon is activated in the presence of a polyamine through the CbrACbrB two-component regulatory proteins (Lu et al., 2002; Nishijyo et al., 2001).

The functions of the SpuA and SpuB enzymes are obscure. However, the impaired growth of $s p u A$ and $s p u B$ mutants on spermidine and spermine suggests that the SpuA and SpuB enzymes play major roles in the catabolism of these polyamines. In addition, $P$. aeruginosa expresses spermidine dehydrogenase (SpdH; EC 1.5.99.6), which has also been identified in Citrobacter freundii and Serratia marcesens (Hisano et al., 1990, 1992a; Tabor \& Kellogg, 1970). The $\mathrm{SpdH}$ of $P$. aeruginosa IFO3080 is constitutively expressed and has been purified as a $63 \mathrm{kDa}$ monomer, but it remains to be characterized in detail (Hisano et al., 1990). In contrast, C. freundii and S. marcesens SpdHs are haem proteins that are inducible by spermidine and they both cleave spermidine to yield 1,3-diaminopropane and 4-aminobutyraldehyde (Fig. 1) and prefer potassium ferricyanide as an electron acceptor (Hisano et al., 1992a, b; Tabor \& Kellogg, 1970). The S. marcescens SpdH oxidizes spermine at an unknown scissile site, but to only about $30 \%$ of its specific 




Succinic semialdehyde

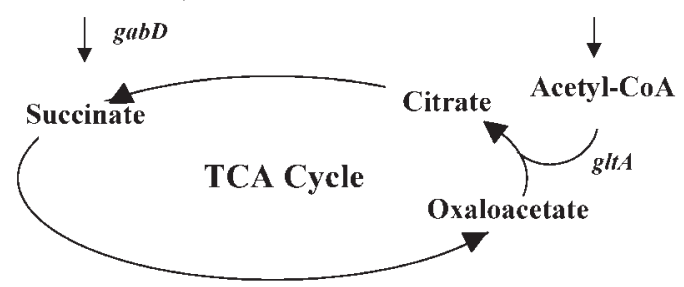

Fig. 1. Reactions catalysed by $\mathrm{SpdH}$ and possible catabolic pathways of reaction products in $P$. aeruginosa PAO1. Reactions catalysed in vitro by $\mathrm{SpdH}$ are indicated by dashed arrows and enzyme genes are assigned to each reaction according to the Pseudomonas Genome Project (http://www.pseudomonas. com): spdH (PA3713), spermidine dehydrogenase; spuC, putrescine aminotransferase, kauB, 3-aminopropanaldehyde/4aminobutyraldehyde/4-guanidinobutyraldehyde dehydrogenase; gabT, 4-aminobutyrate aminotransferase; gabD, succinic semialdehyde dehydrogenase; $g / t A$, citrate synthase. PA0132 and $P A 0130$ might encode $\beta$-alanine aminotransferase and malonic semialdehyde dehydrogenase, respectively. The enzyme catalysing 1,3-diaminopropane deamination to 3-aminopropanaldehyde (dotted arrow) is unknown.

activity for spermidine (Tabor \& Kellogg, 1970). The spdH gene has not been identified in any bacterium.

We purified and characterized the $\mathrm{SpdH}$ of $P$. aeruginosa PAO1 in the present study and then identified the corresponding $s p d H$ gene. Growth experiments with an $s p d H$ knockout mutant and a spuA mutant harbouring the $s p d H$ gene fused to the polyamine-inducible promoter for the $s p u$ operon showed that SpdH can catabolize spermidine in vivo. However, SpdH minimally participates in polyamine utilization by strain PAO1, because expression levels of the enzyme are too low to switch on the catabolic function.

\section{METHODS}

Strains, plasmids and media. We used the described Escherichia coli strains, Pseudomonas aeruginosa PAO1 (wild-type), PAO6100 (kauB::Tn5-751) and PAO5006 (spuA::Tc) and plasmids (Hoang et al., 1998; Jann et al., 1988; Nakada \& Itoh, 2002; Lu et al., 2002; Stover et al., 2000). The P. aeruginosa strains TH2768, TH2774, TH2834, TH3990, TH3992, TH4239 and TH4240 were gifts from N. Gotoh, Department of Microbiology, Kyoto Pharmaceutical University, Kyoto, Japan. P. aeruginosa PAO4548 (spdH:: Gm) was derived from P. aeruginosa PAO1 as described below. The E. coli and
P. aeruginosa strains were cultivated at $37^{\circ} \mathrm{C}$ in Luria-Bertani (LB) and in nutrient yeast broth (NYB), respectively, supplemented with antibiotics when necessary (Haas et al., 1997; Sambrook et al., 1989). Minimal medium P (MMP; Haas et al., 1997) was supplemented with the indicated carbon and nitrogen sources to examine spermidine and spermine utilization and to prepare $P$. aeruginosa cells for assay and for SpdH purification.

DNA manipulations. DNA purification, restriction enzyme analysis and other manipulations proceeded as described previously (Itoh, 1997; Nishijyo et al., 2001). KOD DNA polymerase (Toyobo Biochemicals) was applied to the PCR under the reaction conditions recommended by the supplier and DNA was sequenced using an ABI310 DNA sequencer and Big-Dye Terminator sequencing kits (Applied Biosystems).

Construction of strains and plasmids. To construct an expression plasmid of SpdH and an spdH knockout mutant, we amplified the $s p d H$ coding region by PCR using PAO1 chromosomal DNA as the template and the oligonucleotide primers, 5'-TCATATGACCATCTCCCGCCGCGACTTCC-3' ${ }^{\prime}$ [corresponding to $\overline{\text { nucleotides }+1}$ to +25 relative to the translation initiation codon ATG (bold type) with an NdeI site (underlined) at the $5^{\prime}$ end] and $5^{\prime}$-GTCGCCGTCCTTCAACCCAGTTCGCGGAC-3' (complementary to the $3^{\prime}$-noncoding region between nucleotides +1817 to +1846 from the ATG codon of $s p d H)$. The amplified DNA fragment was cloned into plasmid pUC118 (Vieira \& Messing, 1987) at the HincII site to verify the nucleotides by sequencing. The $1.8 \mathrm{~kb} s p d H$ region in the resultant plasmid, pYI431, was cleaved at the NdeI and HindIII (on the vector plasmid) sites and inserted into pET-22b + (Novagen) between the corresponding restriction sites to produce pYI435. This plasmid was transformed into E. coli BL21(DE3) (Novagen). To construct an $s p d H$ knockout mutant, the $1.8 \mathrm{~kb} s p d H$ segment was excised and cloned as a BamHI-SphI fragment from pYI431 into the mobilizable suicide plasmid pEX18Ap (Hoang et al., 1998) between the corresponding sites, resulting in plasmid pYI432. A gentamicin (Gm)-resistance cassette (Hoang et al., 1998) was inserted as a SmaI fragment into the DraIII site of $s p d H$ on this plasmid to create pYI436. This plasmid was then introduced into strain PAO1 by conjugation via helper E. coli S17-1 (Simon et al., 1983). The spdH:: Gm mutant strain PAO4548 was selected on MMP agar containing $100 \mu \mathrm{g} \mathrm{Gm} \mathrm{ml}^{-1}$ and $20 \mathrm{mM}$ glutamate as the sources of carbon and nitrogen, and then on LB agar containing $5 \%$ sucrose, which allows the growth of clones lost during the second cross-over of the plasmid sequence $\left(\mathrm{sacB}^{+}\right)$integrated into the chromosome (Hoang et al., 1998). To create a $s p u A:: s p d H$ fusion gene, we amplified a $278 \mathrm{bp}$ intergenic region between spuA and spuI ( $\mathrm{Lu}$ et al., 2002) by PCR using oligonucleotide primers designed to add the $N d e$ I sites (underlined) at the translation initiation codons of these genes; 5'-GGGTACCGACATATGGCAACACCTT-3' (nucleotide -13 to +12 of $s p u I$ ) and $5^{\prime}$-CAGGCGAGACATATGACACCCGTTG-3 (complementary to nucleotide -13 to +12 of spuA). After cloning into plasmid pUC118 and verifying the nucleotides as described above, the amplified DNA fragment was isolated as an $N d e I$ fragment and joined to $s p d H$ on plasmid pYI431 at the NdeI site. Because the DNA fragment inserted in the $s p u A:: s p d H$ and spuI::spdH orientations creates Bpu1102I fragments of 709 and $895 \mathrm{bp}$, respectively, we analysed the recombinant plasmids using $B p u 1102$ I restriction endonuclease and identified a plasmid (pYI449) having spuA::spdH. We then digested plasmid pYI449 at the BamHI and HindIII sites (in the multiple cloning sites of pUC118) that flanked the fusion gene and cloned the resultant BamHIHindIII fragment of $s p u A:: s p d H$ between the BglII and HindIII sites of plasmid pNIC6011 (Nakada \& Itoh, 2002), resulting in plasmid pYI451.

SpdH assay. Cells cultured to the exponential phase $\left(\mathrm{OD}_{600} 0 \cdot 6-\right.$ $0 \cdot 8)$ in MMP containing $20 \mathrm{mM}$ glutamate or $20 \mathrm{mM}$ spermidine as 
carbon and nitrogen sources were harvested, washed twice, and then resuspended in $50 \mathrm{mM}$ potassium phosphate buffer $(\mathrm{pH} \mathrm{7 \cdot 2)}$. Cell extracts were prepared by passage through a French pressure cell (SLM-AMINCO) at 20000 p.s.i. (138 MPa), followed by centrifugation at $10000 \mathrm{~g}$ for $30 \mathrm{~min}$ at $4{ }^{\circ} \mathrm{C}$ to remove intact cells and cell debris. Optionally, cytoplasm and cytoplasmic membrane/peptidoglycan fractions were separated by centrifugation at $100000 \mathrm{~g}$ for $1 \mathrm{~h}$ at $4{ }^{\circ} \mathrm{C}$. SpdH activity was determined in a reaction mixture (2 ml) containing $0.5 \mathrm{mM}$ spermidine or spermine, $1.0 \mathrm{mM}$ $\mathrm{K}_{3} \mathrm{Fe}(\mathrm{CN})_{6}$ and $200 \mathrm{mM}$ Tris/ $\mathrm{HCl}$ buffer $\left(\mathrm{pH} \mathrm{7 \cdot 2)}\right.$ at $25^{\circ} \mathrm{C}$. The reaction was initiated by adding a cell-free extract and the decrease in absorbance at $400 \mathrm{~nm}$ (ferricyanide reduction) was measured. One unit of SpdH activity was defined as described (Hisano et al., 1990). Protein concentrations were determined by the method of Bradford (1976) using the Bio-Rad Protein Assay kit, with bovine serum albumin as the standard.

Purification of SpdH from strain PA01. We prepared cell extracts as described above from $P$. aeruginosa PAO1 cells cultured at $37^{\circ} \mathrm{C}$ in MMP (5 l) containing $20 \mathrm{mM}$ glutamate as carbon and nitrogen sources. SpdH was precipitated with ammonium sulphate (40-60\% saturation) dissolved in, and exhaustively dialysed against, $50 \mathrm{mM}$ potassium phosphate buffer $(\mathrm{pH} 7 \cdot 2)$. The dialysed enzyme was eluted through a Hiprep16/10 DEAE column (20 ml; Amersham Biosciences) using a linear gradient $(0-1 \cdot 0 \mathrm{M})$ of $\mathrm{KCl}$ in $50 \mathrm{mM}$ potassium phosphate buffer $(\mathrm{pH} \mathrm{7 \cdot 2)}$, with which the column had been equilibrated. The active fractions $(5.0 \mathrm{ml}$ each, eluted at $0.03 \mathrm{M} \mathrm{KCl}$ ) were pooled, dialysed against the potassium phosphate buffer and applied to a MonoQ 5/50GL column ( $1 \cdot 0 \mathrm{ml}$; Amersham Biosciences) equilibrated with $50 \mathrm{mM}$ potassium phosphate buffer $(\mathrm{pH} 8 \cdot 0)$. The enzyme was eluted with a $\mathrm{KCl}$ linear gradient $(0$ $1.0 \mathrm{M})$ in the same buffer and the active fractions $(0.6 \mathrm{ml}$ each, eluted at $0.35 \mathrm{M} \mathrm{KCl}$ ) were further purified by gel filtration through a column containing Superose12 HR 10/30 (Amersham Biosciences) using $50 \mathrm{mM}$ potassium phosphate buffer $(\mathrm{pH} \mathrm{7 \cdot 2)}$ containing $150 \mathrm{mM} \mathrm{KCl}$ as the elution buffer. The enzyme purity was examined by SDS-PAGE (Nakada \& Itoh, 2002).

Expression and purification of SpdH in E. coli. We expressed SpdH in E. coli BL21(DE3) harbouring plasmid pYI435 (see above). IPTG $(1 \mathrm{mM})$ was added to the recombinant culture growing exponentially $\left(\mathrm{OD}_{600} 0 \cdot 3\right)$ in LB medium to induce $\mathrm{SpdH}$ synthesis. After $4 \mathrm{~h}$ incubation the cells were collected by centrifugation, suspended in $50 \mathrm{mM}$ potassium phosphate buffer $(\mathrm{pH} \mathrm{7 \cdot 2)}$ and ruptured by passage through an Amicon French press. Cell-free extract was obtained by centrifugation at $15000 \mathrm{~g}$ for $30 \mathrm{~min}$ and recombinant $\mathrm{SpdH}$ was purified by chromatography using columns containing Hiprep16/10 DEAE, MonoQ 5/50GL and Superose12 HR $10 / 30$ as described above.

HPLC analysis of polyamines and their reaction products. To determine the reaction products of spermidine and spermine, we incubated the polyamines with purified $\mathrm{SpdH}$ in the assay mixture $(2 \mathrm{ml})$ as described above. Samples $(0.25 \mathrm{ml})$ were removed at the indicated times and the reaction was terminated by adding $1 \mathrm{M} \mathrm{HCl}$ $\left(20 \mu \mathrm{l} \mathrm{ml}^{-1}\right)$. Insoluble materials were removed by passage through a cellulose acetate membrane (pore size $0.45 \mu \mathrm{m}$; Advantec). Thereafter, portions $(20 \mu \mathrm{l})$ were eluted through a Shodex Asahipak ODP-50 4E column $(4 \cdot 6 \times 250 \mathrm{~mm}$; Asahi Chemical Industry) using $200 \mathrm{mM}$ sodium phosphate buffer $(\mathrm{pH} 7 \cdot 7)$ containing $2 \cdot 3 \mathrm{mM}$ sodium 1-octane sulphonate as the mobile phase to resolve spermidine and its products, or through a Cadenza CD-C18 column $(4.6 \times 250 \mathrm{~mm}$; Imtakt $)$ to separate spermine and its products using $30 \%(\mathrm{v} / \mathrm{v})$ acetonitrile as the mobile phase. The elution rate was $0.5 \mathrm{ml} \mathrm{min}{ }^{-1}$. The substrates and their products eluted from the HPLC columns were coupled with o-phthalaldehyde and detected by measuring emission at $455 \mathrm{~nm}$ (excision at $345 \mathrm{~nm}$ ) using a RF-10AXL fluorescence detector (Shimadzu) and an amino acid detection system OPA (Shimadzu). We used the same HPLC columns under conditions identical to those applied to spermidine and spermine determinations in cultures. Wild-type PAO1 and spdH mutant cells $\left(1 \times 10^{9} \mathrm{ml}^{-1}\right)$ growing exponentially in MMP containing $20 \mathrm{mM}$ spermidine (inducible substrate) or in $20 \mathrm{mM}$ glutamate (non-inducible substrate) as the sole carbon and nitrogen sources were transferred to fresh MMP $(10 \mathrm{ml})$ containing $20 \mathrm{mM}$ spermidine or $20 \mathrm{mM}$ spermine and shaken at $37^{\circ} \mathrm{C}$. Duplicate portions $(0.2 \mathrm{ml})$ of the cultures were removed at the indicated time. The cells were sedimented by centrifugation and the supernatant was passed through cellulose acetate membranes. Thereafter, portions $(20 \mu \mathrm{l})$ of the filtrate were eluted through the respective HPLC columns. Spermidine, spermine and 1,3-diaminopropane were purchased from Sigma and 4-aminobutyraldehyde and 3-aminopropanaldehyde were synthesized as described previously (Tanaka et al., 2001; Yorifuji et al., 1986).

Amino acid sequencing and MALDI-TOF mass spectrometry. Purified SpdH was resolved by $10 \%$ SDS-PAGE and blotted onto a PVDF membrane (Amersham Biosciences). The amino-terminal sequence was determined using an HP10000 protein sequencer (Hewlett Packard). We measured the MALDI-TOF mass spectra of purified SpdH using a REFLEX II equipped with a SCOUT ion source and a pulsed ion extraction accessory (Bruker Daltonics). Protein samples were mixed with the same volume of a matrix solution $(1 \%(\mathrm{w} / \mathrm{v})$ of 3,5-dimethoxy-4-hydroxycinnamic acid (Bruker Daltonics) in acetonitrile/ $/ 1 \%$ trifluoroacetic acid $(1: 2, \mathrm{v} / \mathrm{v})$ and pulse-irradiated with a nitrogen laser at $337 \mathrm{~nm}$ for $3 \mathrm{~ns}$ each set in the linear mode at $20 \mathrm{kV}$ of ion acceleration. Spectra were the means of at least 100 laser shots.

\section{RESULTS}

\section{Occurrence and constitutive synthesis of SpdH among $\boldsymbol{P}$. aeruginosa strains}

Unlike S. marcescens ATCC 25179 and C. freundii IFO12681, which synthesize 6- and 20-fold more SpdH, respectively, in the presence of spermidine, $P$. aeruginosa IFO3080 constitutively produces this enzyme (Hisano et al., 1990, 1992b; Tabor \& Kellogg, 1970). To determine whether other $P$. aeruginosa strains also have $\mathrm{SpdH}$ and if so, whether they constitutively produce the enzyme, we cultured strain PAO1 and seven other independent $P$. aeruginosa strains in MMP containing $20 \mathrm{mM}$ spermidine (inducing substrate) or $20 \mathrm{mM}$ glutamate (non-inducing substrate) as the sole sources of carbon and nitrogen, and measured $\mathrm{SpdH}$ activities in the cell extracts. All tested strains formed similar amounts of the enzyme [about 0.02 units $(\mathrm{mg} \text { protein })^{-1}$ ] irrespective of the presence of the inducer, providing evidence that $\mathrm{SpdH}$ occurs universally and is constitutively synthesized among $P$. aeruginosa strains. Approximately half the enzymes were associated with the cell envelope (sediment after centrifugation at $100000 \mathrm{~g}$ for $60 \mathrm{~min}$ ) of all tested strains.

\section{Identification of $\mathbf{s p d H}$}

As described in Methods, we purified SpdH 2100-fold from PAO1 cells with a yield of $2.6 \%(2 \mu \mathrm{g}$ protein from 11 culture). Separation by SDS-PAGE showed that the purified enzyme contained 69 and $47 \mathrm{kDa}$ proteins (see 
Supplementary Fig. S1, available with the online version of this paper). Because the molecular mass of SpdH determined by gel-filtration chromatography (see Methods) was $70 \mathrm{kDa}$, we sequenced the first 15 amino-terminal residues (APGGRYYPPALTGLR) of the $69 \mathrm{kDa}$ protein. A BLAST P search using this amino-terminal sequence against the protein sequences in the Pseudomonas genome database (http:// www.pseudomonas.com) identified the PA3713 gene, which encodes a protein with the same sequence between residues 29 and 43. The calculated $M_{\mathrm{r}}$ (65 907) of mature PA3713 protein (29-620 residues) is in agreement within experimental error with the determined $M_{\mathrm{r}}$ of $\mathrm{SpdH}$, and the PA3713 protein expressed in E. coli displayed SpdH activity (see below), substantiating the notion that PA3713 is the gene encoding SpdH. We hereinafter refer to $P A 3713$ as $s p d H$. This gene is also present in $P$. aeruginosa strains 2192 (accession no. ZP_00973472), C3719 (ZP_00967906) and UCBPP-PA14 (ZP_00137108), the genome sequences of which have been determined. However, the gene is absent in closely related Pseudomonas species such as Pseudomonas fluorescens, Pseudomonas putida and Pseudomonas syringae. Homologues of SpdH ( $\geqslant 43 \%$ identity and $\geqslant 56 \%$ similarity) occur in a few beta- and epsilonproteobacterial species, including Chromobacterium violaceum (Vasconcelos et al., 2003), Campylobacter lari (Fouts et al., 2005), Campylobacter jejuni (accession no. ZP_01099292), Ralstonia metallidurans (AAAI03000006.1), Ralstonia eutropha (AAZ60824) and culicine Anopheles gambiae (AAAB01002848.1).

\section{Purification and characterization of SpdH expressed in $E$. coli}

To confirm that $s p d H$ encodes SpdH and to characterize the enzyme, we cloned the coding DNA region into the plasmid pET-22b $(+)$ between the NdeI and HindIII sites, so that the cloned gene was transcribed from the T7 promoter on the expression plasmid. The resultant plasmid pYI435 was introduced into E. coli BL21(DE3) that carries the IPTGinducible T7 RNA polymerase gene. Cultivation of the recombinant $E$. coli in the presence of $1 \mathrm{mM}$ IPTG induced sufficient SpdH synthesis to produce $7 \cdot 0$ units (mg protein $)^{-1}$ of the enzyme, which was about 300 -fold more than the amount produced by strain PAO1. Three column chromatography separations (see Methods) yielded $4 \mathrm{mg}$ apparently homogeneous SpdH per litre of culture, as judged by SDS-PAGE (see Fig. S1, lane 2). The first 16 amino-terminal residues of the purified enzyme were TISRRDFLNGVALTI. This sequence precisely matched the deduced amino-terminal sequence except for the first methionine, which appeared to be post-translationally eliminated. The signal peptide was not cleaved in the E. coli host. Presumably a signal peptidase of the heterologous host cannot recognize this sequence, or the abundant synthesized $s p d H$ mRNA is translated in the cytoplasm by free ribosomes.

Absorption peaks of purified SpdH appeared at 280 and $415 \mathrm{~nm}$ in the oxidized state. In the reduced state (in the presence of substrate), enzyme absorption peaked at 340 , 530 and $560 \mathrm{~nm}$. These spectrometric profiles are typical of haem proteins and are very similar to those observed with S. marcescens and C. freundii SpdHs (Hisano et al., 1992b; Tabor \& Kellogg, 1970). The molecular mass of the recombinant SpdH determined by MALDI-TOF-mass spectrometry was 69850 . This value is larger than the calculated $M_{\mathrm{r}}$ (68730) by $1120 \mathrm{Da}$. We consider possible prosthetic groups of $\mathrm{SpdH}$ in the Discussion. The purified SpdH preferentially used ferricyanide as an electron acceptor. Phenazine methosulphate and dichloroindophenol also served as electron acceptors but at about $10 \%$ of the efficiency of ferricyanide. Cytochrome $c$ was a poor electron acceptor for this enzyme ( $2 \%$ ferricyanide). Measurements of initial reaction velocities with different substrate concentrations determined the specific activity and the $K_{\mathrm{m}}$ value of the enzyme for spermidine as $37 \pm 2$ units ( $\mathrm{mg}$ protein) $)^{-1}$ and $36 \pm 2 \mu \mathrm{M}$, respectively. The enzyme also used spermine as a substrate. The specific activity towards spermine was $25 \pm 2$ units (mg protein) $)^{-1}$ and the $K_{\mathrm{m}}$ value for this polyamine was $18 \pm 1 \mu \mathrm{M}$.

\section{Cleavage modes on spermidine and spermine}

We next investigated where SpdH cleaves spermidine and spermine using HPLC analysis of the reaction products. This enzyme yielded mostly 1,3-diaminopropane and 4-aminobutyraldehyde from spermidine and trace amounts of 3-aminopropanaldehyde (Fig. 2A). Thus, like S. marcesens and C. freundii SpdHs (Hisano et al., 1992b; Tabor \& Kellogg, 1970), P. aeruginosa SpdH selectively cleaves the $\mathrm{N}$-4-aminobutylimino bond of spermidine. This enzyme generated 3-aminopropanaldehyde and spermidine from spermine during the initial stage of the reaction, and the produced spermidine was decomposed into 1,3-diaminopropane and 4-aminobutyraldehyde at a later stage of the reaction (Fig. $2 \mathrm{~B}$ ), showing that $P$. aeruginosa $\mathrm{SpdH}$ cleaves spermine at the $\mathrm{N}$-3-aminopropylimino bonds.

\section{Growth phenotypes of spdH and kauB mutants}

To determine whether $P$. aeruginosa PAO1 SpdH contributes to spermidine and spermine utilization in any way, we inactivated $s p d H$ by inserting a Gm cassette (Hoang et al., 1998) into the DraIII site at nucleotide 1117 of $s p d H$ (1863 bp). The resultant spdH mutant, strain PAO4548 (spdH:: Gm), had no SpdH activity either in the membranes or in the cytoplasm, indicating that both enzymes are the $s p d H$ product. Possibly SpdH dissociates from the membranes during cell disruption. The $s p d H:: \mathrm{Gm}$ strain proliferated as normally as wild-type PAO1 in MMP supplemented with $20 \mathrm{mM}$ spermidine or spermine as the sole source of both carbon and nitrogen (data not shown). The spdH mutant also grew normally in MMP containing glutamate as the sole source of carbon and nitrogen, or succinate and ammonia. To examine whether $\mathrm{SpdH}$ participates at any level in polyamine catabolism in vivo, we cultivated strains PAO1 (wild-type) and PAO4548 (spdH:: Gm) cells in MMP containing $20 \mathrm{mM}$ glutamate 
A



B

$0 \mathrm{sec}$

$\mathrm{e}$

10
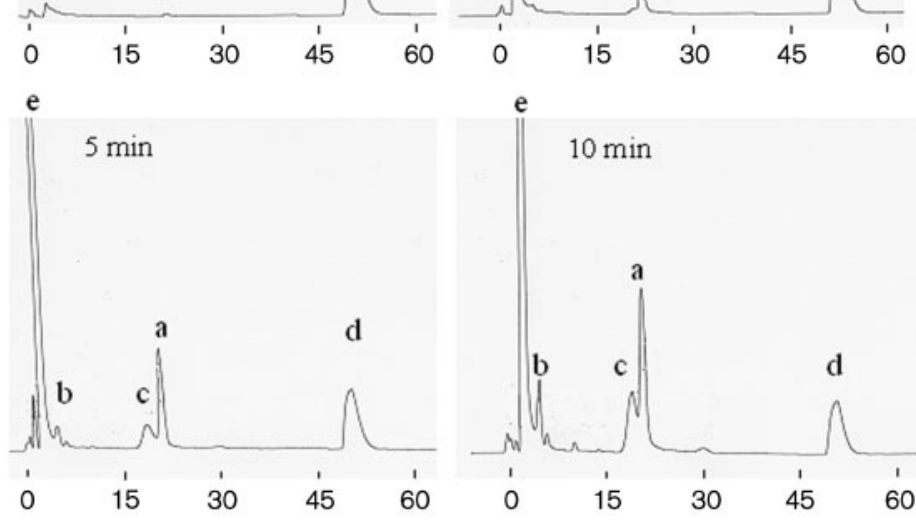

Retention time ( $\mathrm{min})$

Fig. 2. HPLC analysis of products of reactions between spermidine (A) and spermine (B) with SpdH. Reaction mixtures were sampled at the indicated times and analysed by HPLC. Retention times of substrates and products were determined using commercial or synthesized compounds: a, spermidine; b, 1,3-diaminopropane; c, 4-aminobutyraldehyde; $d$, spermine; e, 3-aminopropanaldehyde. Fluorescence emission intensity differs depending on the compound and hence does not reflect chemical amounts.

or $20 \mathrm{mM}$ spermidine to the exponential growth phase and transferred the induced and non-induced cells into fresh MMP containing $20 \mathrm{mM}$ spermidine or $20 \mathrm{mM}$ spermine. The polyamine amounts in the cultures determined by HPLC showed that the non-induced cells of both strains utilized the polyamines very slowly and at comparable rates (see Supplementary Fig. S2, available with the online version of this paper). The induced cells, regardless of the $s p d H$ mutation, actively and again similarly consumed both the polyamines. Spermidine was utilized three times faster than spermine (see Fig. S2) and hence is a more efficient source of both carbon and nitrogen than spermine (see below). Thus, SpdH contributed little to polyamine catabolism by $P$. aeruginosa $\mathrm{PAO} 1$ under our growth conditions

The kauB gene encodes an aldehyde dehydrogenase, which catalyses the oxidative conversion of 4-guanidinobutyraldehyde to 4-guanidinobutyrate in the arginine dehydrogenase pathway and of 4-aminobutyradehyde to 4-aminobutyrate in the putrescine catabolic pathway. The mutation of $k a u B$ therefore simultaneously abolishes the utilization of 2ketoarginine and putrescine (Jann et al., 1988). We found that the kauB mutant strain PAO6100 (kauB::Tn5-751) (Jann et al., 1988) also could not grow on any of spermidine, spermine, or 1,3-diaminopropane.

\section{In vivo catabolic function of SpdH}

Although $P$. aeruginosa $\mathrm{PAO} 1 \mathrm{SpdH}$ actively degrades spermidine and spermine in vitro, it seemed not to be involved significantly in their catabolism and utilization. Perhaps the constitutive levels of $\mathrm{SpdH}$, being below $5 \%$ of the induced levels in C. freundii (Hisano et al., 1990), are insufficient to do so. To demonstrate a catabolic function of $P$. aeruginosa SpdH in vivo, we fused in-frame the $s p d H$ structural gene to the polyamine-inducible promoter of $s p u A$ at the translation initiation codons of these genes and cloned the resultant spuA::spdH chimeric gene into plasmid pNIC6011 (Nishijyo et al., 2001), to create pYI451 (see Methods). When this plasmid was introduced into strain PAO5006 (spuA::Tc), which grows poorly on either spermidine (Lu et al., 2002; Fig. 3) or spermine (data not shown), the spuA mutant initiated growth in MMP + $20 \mathrm{mM}$ spermidine much earlier than wild-type PAO1 and proliferated at a growth rate (doubling time of about $110 \mathrm{~min}$ ) similar to that of the wild-type strain (Fig. 3). PAO1, PAO5006 and PAO5006/pNIC6011 cells growing in $\mathrm{MMP}+20 \mathrm{mM}$ glutamate or $20 \mathrm{mM}$ spermidine produced approximately $0 \cdot 02$ units $\mathrm{SpdH}(\mathrm{mg} \text { protein })^{-1}$. PAO5006/ pYI451 cells contained 0.07 units SpdH (mg protein $)^{-1}$ during growth in $\mathrm{MMP}+20 \mathrm{mM}$ glutamate. However, 


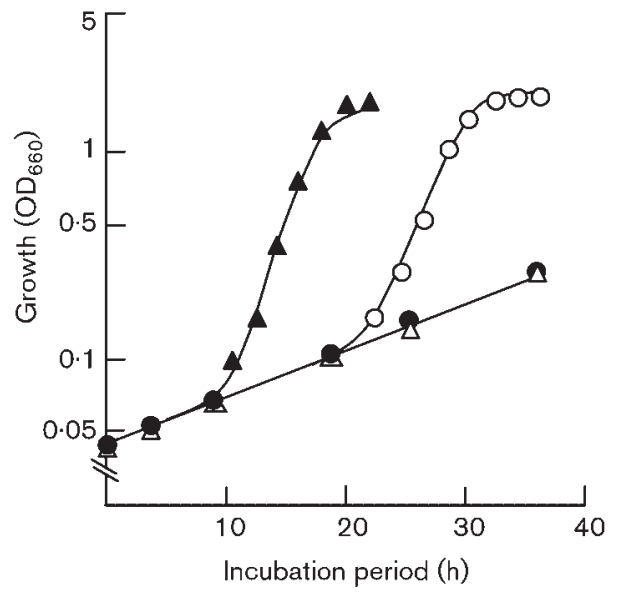

Fig. 3. Catabolic function of $\mathrm{SpdH}$. Strains were incubated in MMP containing $20 \mathrm{mM}$ spermidine at $37^{\circ} \mathrm{C}$ and growth was followed by measuring optical density at $660 \mathrm{~nm}$. $\bigcirc, P$. aeruginosa PAO1; ๑, P. aeruginosa PAO5006 (spuA::Tc); $\square, P$. aeruginosa PAO5006/pNIC6011; $\Delta, P$. aeruginosa PAO5006/ pYI451 (spuA:: spdH).

when incubated in MMP containing $20 \mathrm{mM}$ spermidine the recombinant cells induced SpdH synthesis to form $0 \cdot 24$ units $(\mathrm{mg} \text { protein })^{-1}$, amounts that were fairly similar to those generated $\left(0 \cdot 2-0 \cdot 6\right.$ units $\left.(\mathrm{mg} \text { protein })^{-1}\right)$ in the induced C. freundii and S. marcescens (Hisano et al., 1990; Tabor \& Kellogg, 1970). Plasmid pYI451 did not support growth of the spuA mutant on spermine. Furthermore, $P$. aeruginosa $\mathrm{PAO} 1$ grew much more slowly (doubling time of $150 \mathrm{~min}$ ) in MMP containing $20 \mathrm{mM}$ spermine than in that containing spermidine. Spermine might not effectively induce the spuA promoter.

\section{DISCUSSION}

P. aeruginosa $\mathrm{PAO} 1 \mathrm{SpdH}$ shares similar properties with its S. marcescens and C. freundii counterparts (Hisano et al., 1990; Tabor \& Kellogg, 1970). These are monomers of haem proteins associated with cytoplasmic membranes that cleave spermidine into 1,3-diaminopropane and 4-aminobutyraldehyde and preferentially use ferricyanide as an electron acceptor in vitro. Like S. marcescens SpdH (Okada et al., 1979; Tabor \& Kellogg, 1970), the P. aeruginosa enzyme can also cleave spermine, but at a different scissile site from the $S$. marcescens enzyme. Spermidine and 3-aminopropanaldehyde are the sole products of spermine during the initial stage of the reaction (Fig. 2B); 1,3-diaminopropane becomes detectable only after spermidine has accumulated and the amount of the diamine increases in parallel with an increase in 4-aminobutyradehyde (Fig. 2B). These profiles of the reaction products show that the diamine is derived from the product spermidine and not the initial substrate, spermine. Thus $P$. aeruginosa $\mathrm{SpdH}$ specifically acts on the propylimino bonds of spermine, whereas $S$. marcescens SpdH preferentially cleaves spermine at the butylimino bonds, yielding 1,3-diaminopropane and $\mathrm{N}$-3-aminopropionyl-4-aminobutyraldehyde (Okada et al., 1979).

The SpdH of S. marcescens is a flavoprotein and that of $C$. freundii is a quinoprotein (Hisano et al., 1992a; Tabor \& Kellogg, 1970). The P. aeruginosa SpdH does not appear to be a quinoprotein, because it has no sequence similarity to known quinoproteins that have a conserved pyrroloquinoline quinone (PQQ)-binding domain and a homologous haem C-binding sequence in the carboxyl-terminal region (Oubrie, 2003; Yamada et al., 2003). The SpdH of P. aeruginosa has a pfam_fs:FAD_binding_2-FAD binding domain (N-score, 14 , 35; E-value, $9 \cdot 4 \mathrm{e}^{-08}$ ) and the pfam_fs: DAO motif ( $\mathrm{N}$-score, 15, 33; E-value, $1 \mathrm{e}^{-08}$ ) of FAD-dependent oxidoreductase (http://kr.expasy.org/prosite) between residues 75 and 114. The carboxyl-terminal region (520-620 residues) contains the pfam_fs:Amino_oxidase motif (Nscore, 12, 39; E-value, $8 \cdot 6 \mathrm{e}^{-\overline{06}}$ ) of an amine oxidoreductase that contains flavin. These motifs are conserved in oxidoreductases containing flavin such as glycine/D-amino acid oxidase (Mattevi et al., 1997; Mortl et al., 2004) and FixC dehydrogenase (Edgren \& Nordlund, 2004). The predicted molecular mass of the prosthetic groups $\left(M_{\mathrm{r}} 1120\right)$ is close to the combined molecular mass of haem $\left(M_{\mathrm{r}} \sim 620\right)$ and FMN $\left(M_{\mathrm{r}} 456\right)$. The structure of the prosthetic flavin should be confirmed by chemical analysis. The SpdH purified from $\mathrm{PAO} 1$ cells is truncated at the amino terminal by 28 residues. This region has features typical of a signal peptide (peak value, $2 \cdot 10$; an amino-terminal basic region with a net charge of 1 , a central hydrophobic region of 16 residues and a discriminant score of 2.03 ) according to the PSORT program (http://psort.ims.u-tokyo.ac.jp). The TMpred program (http://www.ch.embnet.org) predicted a short transmembrane segment (score of 1661) between residues 76 and 92. These features of the $\mathrm{SpdH}$ sequence are consistent with the membrane location of the enzyme and account for weak interaction with the cytoplasmic membranes, since sonication, low ionic concentration buffers and detergents can dissociate SpdH from membranes.

SpdH appears to occur universally and be constitutively expressed at low levels in $P$. aeruginosa strains. Knockout of $s p d H$ did not apparently affect growth on spermidine of $P$. aeruginosa $\mathrm{PAO}$, because this strain has the $s p u A B$ genes that encode inducible enzymes for spermidine catabolism (Lu et al., 2002). However, a knockout mutant of spuA can still grow, though slowly, on spermidine (Lu et al., 2002; Fig. 3), suggesting that $s p d H$ contributes to this slow growth of the spuA mutant. When SpdH is expressed in vivo in appropriate amounts, this enzyme can fully support growth on spermidine of a spuA mutant (Fig. 3), demonstrating the in vivo catabolic function of this enzyme. 4-Aminobutyraldehyde, one of the degradation products of spermidine by $\mathrm{SpdH}$, would be converted by $\mathrm{KauB}$ aldehyde dehydrogenase to 4 -aminobutyrate, which is then led to succinate via 4-aminobutyrate aminotransferase (GabT or GoaD) and succinic semialdehyde dehydrogenase (GabD) (http://www.pseudomonas.com) as in E. coli 
(Kurihara et al., 2005; Schneider et al., 2002) (Fig. 1). The other product, 1,3-diaminopropane, can serve as a source of both carbon and nitrogen for $P$. aeruginosa PAO1. In Arthrobacter spp. an aminotransferase converts 1,3-diaminopropane along with pyruvate into 3-aminopropanaldehyde and alanine (Yorifuji et al., 1997). The catabolic enzymes of this diamine have not been studied in $P$. aeruginosa. However, the kauB mutation abolished growth on 1,3-diaminopropane, suggesting that $P$. aeruginosa $\mathrm{PAO} 1$ also catabolizes the diamine via 3 -aminopropanaldehyde, which is then oxidized to $\beta$-alanine by $\mathrm{KauB}$ dehydrogenase (Fig. 1). According to the Pseudomonas Genome Project (http://www.pseudomonas.com), PA0132 and PA0130 are good candidates for $\beta$-alanine aminotransferase and malonic semialdehyde dehydrogenase, respectively (Fig. 1). P. aeruginosa $\mathrm{PAO} 1$ thus appears to have all the enzymes required to catabolize the compounds yielded from spermidine and spermine by SpdH.

Since the inactivation of $s p u A$ and $s p u B$ results in defective spermidine (Lu et al., 2002) and spermine (data not shown) utilization, the products of these genes should play key roles in polyamine catabolism. Kurihara et al. (2005) recently reported that $E$. coli converts putrescine by YcjK to $N-\gamma$ glutamylputrescine, which is subsequently oxidatively deaminated to $\gamma$-glutamylaminobutyraldehyde that is in turn oxidized to $\gamma$-glutamylaminobutyrate. YcjL hydrolyses $\gamma$-glutamylaminobutyrate into glutamate and 4 -aminobutyrate. SpuA and SpuB share similar amino acid sequences with YcjL (60\% similarity) and YcjK (50\% similarity), respectively. Such similarity and the impaired growth of a $k a u B$ mutant on spermidine and spermine imply that the catabolism of these polyamines in $P$. aeruginosa proceeds via $\gamma$-glutamyl intermediates and that KauB aldehyde dehydrogenase is involved in the oxidation of intermediate aldehydes. We are presently analysing the catabolic intermediates that accumulate in $s p u A B$ and $k a u B$ mutants to establish the catabolic routes of spermidine and spermine in $P$. aeruginosa $\mathrm{PAO}$.

\section{ACKNOWLEDGEMENTS}

We thank D. Haas, C.-D. Lu and P. Schweizer for providing strain PAO6100, strain PAO5006 and plasmid pEX18Ap, respectively. We are also grateful to N. Gotoh for the gifts of strains TH2768 to TH4240. This study was supported in part by a grant-in-aid for scientific research from the Japan Society for the Promotion of Sciences (JSPS) to Y.I. (14360060). V.V.D. and Y.N. were research fellows supported by JSPS.

\section{REFERENCES}

Bradford, M. M. (1976). A rapid and sensitive method for the quantitation of microgram quantities of protein utilizing the principle of protein-dye binding. Anal Biochem 72, 248-254.

Edgren, T. \& Nordlund, S. (2004). The fixABCX genes in Rhodospirillum rubrum encode a putative membrane complex participating in electron transfer to nitrogenase. J Bacteriol 186, 2052-2060.
Fouts, D. E. \& 20 other authors (2005). Major structural differences and novel potential virulence mechanisms from the genome of multiple Campylobacter species. PLoS Biol 3, 72-85.

Haas, D., Holloway, B. W., Schamböck, A. \& Leisinger, T. (1997). The genetic organism of arginine biosynthesis in Pseudomonas aeruginosa. Mol Gen Genet 154, 7-22.

Hisano, T., Abe, S., Wakashiro, M., Kimura, A. \& Murata, K. (1990). Microbial spermidine dehydrogenase: purification and properties of the enzyme in Pseudomonas aeruginosa and Citrobacter freundii. $J$ Ferment Bioeng 69, 335-340.

Hisano, T., Murata, K., Kimura, A., Matsushita, K. \& Adachi, O. (1992a). Further properties of spermidine dehydrogenase from Citrobacter freundii IFO 12681. Biosci Biotechnol Biochem 56, 311-314.

Hisano, T., Murata, K., Kimura, A., Matsushita, K., Toyama, H. \& Adachi, O. (1992b). Characterization of membrane-bound spermidine dehydrogenase of Citrobacter freundii. Biosci Biotechnol Biochem 56, 1916-1920.

Hoang, T. T., Karkhoff-Schweizer, R. R., Kutchma, A. J. \& Schweizer, H. P. (1998). A broad-host-range Flp-FFT recombination system for site-specific excision of chromosomally-located DNA sequence: application for isolation of unmarked Pseudomonas aeruginosa mutants. Gene 212, 77-86.

Itoh, Y. (1997). Cloning and characterization of the aru genes encoding enzymes of the catabolic arginine succinyltransferase pathway in Pseudomonas aeruginosa PAO1. J Bacteriol 179, 7280-7290.

Jann, A., Matsumoto, H. \& Haas, D. (1988). The fourth arginine catabolic pathway of Pseudomonas aeruginosa. J Gen Microbiol 134, 1043-1053.

Kurihara, S., Oda, S., Kato, K., Kim, H. G., Koyanagi, T., Kumagai, H. \& Suzuki, H. (2005). A novel putrescine utilization pathway involves $\gamma$-glutamylated intermediates of Escherichia coli K-12. J Biol Chem 280, 4602-4608.

Lu, C. D., Itoh, Y., Nakada, Y. \& Jiang, Y. (2002). Functional analysis and regulation of the divergent spuABCDEFGH-spuI operons for polyamine uptake and utilization in Pseudomonas aeruginosa PAO1. J Bacteriol 184, 3765-3773.

Mattevi, A., Vanoni, M. A. \& Curti, B. (1997). Structure of D-amino acid oxidase: new insights from an old enzyme. Curr Opin Struct Biol 7, 804-810.

Mortl, M., Diederichs, K., Welte, W., Molla, G., Motteran, L., Andriolo, G., Pilone, M. S. \& Pollegioni, L. (2004). Structurefunction correlation glycine oxidase from Bacillus subtilis. $J$ Biol Chem 279, 29718-29727.

Nakada, Y. \& Itoh, Y. (2002). Characterization and regulation of the $g b u \mathrm{~A}$ gene, encoding guanidinobutyrase in the arginine dehydrogenase pathway of Pseudomonas aeruginosa PAO1. J Bacteriol 184, 3377-3384.

Nishijyo, T., Haas, D. \& Itoh, Y. (2001). The $c b r A-c b r B$ two component regulatory system controls the utilization of multiple carbon and nitrogen sources in Pseudomonas aeruginosa. Mol Microbiol 40, 917-931.

Okada, M., Kawashima, S. \& Imahori, K. (1979). Substrate binding characteristics of the active site of spermidine dehydrogenase from Serratia marcescens. J Biochem 85, 1235-1243.

Oubrie, A. (2003). Structure and mechanism of soluble glucose dehydrogenase and other PQQ-dependent enzymes. Biochim Biophys Acta 1647, 143-151.

Sambrook, J., Fritsch, E. F. \& Maniatis, T. (1989). Molecular Cloning: a Laboratory Manual, 2nd edn. Cold Spring Harbor, NY: Cold Spring Harbor Laboratory.

Schneider, B. L., Ruback, S., Kiupakis, A. K., Kasbarian, H., Pybus, C. \& Reitzer, L. (2002). The Escherichia coli gabDTPC operon: 
specific $\gamma$-aminobutyrate catabolism and nonspecific induction. J Bacteriol 184, 6976-6986.

Simon, R., Priefer, U. \& Pühler, A. (1983). A broad-host range mobilization system for in vivo genetic engineering: transposon mutagenesis in Gram-negative bacteria. Bio/Technology 1, 784-790.

Stanier, R. Y., Palleroni, N. J. \& Doudoroff, M. (1966). The aerobic pseudomonads: a taxonomic study. J Gen Microbiol 43, 159-271.

Stover, C. V., Pham, X. Q., Erwin, A. L., Mizoguchi, S. D., Warrener, P. \& 25 other authors (2000). Complete genome sequence of Pseudomonas aeruginosa PAO1, an opportunistic pathogen. Nature 406, 959-964.

Tabor, C. W. \& Kellogg, P. D. (1970). Identification of flavin dinucleotide and heme in a homogeneous spermidine dehydrogenase from Serratia marcescens. J Biol Chem 245, 5424-5433.

Tanaka, K., Matsuno, E., Shimizu, E., Shibai, H. \& Yorifuji, T. (2001). Purification and characterization of aminopropionaldehyde dehydrogenase from Arthrobacter sp. TMP-1. FEMS Microbiol Lett 195, 191-196.
Vasconcelos, A. T. R. \& 108 other authors (2003). The complete genome sequence of Chromobacterium violaceum reveals remarkable and exploitable bacterial adaptability. Proc Natl Acad Sci U S A 100, 11660-11665.

Vieira, J. \& Messing, J. (1987). Production of single-stranded plasmid DNA. Methods Enzymol 153, 3-11.

Yamada, M., Elias, M. D., Matsushita, K., Migita, C. T. \& Adachi, O. (2003). Escherichia coli PQQ-containing quinoprotein glucose dehydrogenase: its structure comparison with other quinoproteins. Biochim Biophys Acta 1647, 185-192.

Yorifuji, T., Koike, K., Sakurai, T. \& Yokoyama, K. (1986). 4Aminobutyraldehyde and 4-guanidinobutyraldehyde dehydrogenase for arginine degradation in Pseudomonas putida. Agric Biol Chem 50, 2009-2016.

Yorifuji, T., Ishihara, T., Naka, T., Kondo, S. \& Shimizu, E. (1997). Purification and characterization of polyamine aminotransferase of Arthrobacter sp. TMP1. J Biochem 122, 537-543. 